\title{
Review and Current Status of Opisthorchis viverrini Infection at the Community Level in Thailand
}

\author{
Natthawut Kaewpitoon ${ }^{1,2,3 *}$, Nusorn Kootanavanichpong ${ }^{1}$, Ponthip Kompor ${ }^{1}$, \\ Wasugree Chavenkun ${ }^{1}$, Jirawoot Kujapun ${ }^{1}$, Jun Norkaew ${ }^{1}$, Sukanya Ponphimai ${ }^{1}$, \\ Likit Matrakool $^{2}$, Taweesak Tongtawee ${ }^{2}$, Sukij Panpimanmas $^{2}$, Ratana Rujirakul ${ }^{3}$, \\ Natnapa Padchasuwan ${ }^{4}$, Poowadol Pholsripradit ${ }^{5}$, Thawatchai Eksanti ${ }^{6}$, Tanida \\ Phatisena $^{6}$, Ryan A Loyd ${ }^{2,3}$, Soraya J Kaewpitoon ${ }^{2,3}$
}

\begin{abstract}
Opisthorchis viverrini is remains a public health problem in Thailand, particularly in the northeast and north regions which have the highest incidences of chonalgiocarcinoma (CCA). O. viverrini causes the disease opithorchiasis, and its has been classified as a group 1 biological carcinogen. Humans, dogs, and cats become infected with $O$. viverrini by ingesting raw or undercooked fish containing infective metacercariae. The first human cases of $O$. viverrini infection were reported in Thailand 100 years ago, and it's still a problem at the community level. Based on data for the year 2009, more than 6 million people were infected with $O$. viverrini. Associated medical care and loss of wages in Thailand costs about \$120 million annually. This review highlights the current status of $O$. viverrini infection in communities of Thailand through active surveillance for the five years period from 2010 and 2015. A total of 17 community-based surveys were conducted, most in the northeast region. Some 7 surveys demonstrated a high prevalence over $20 \%$, and the highest was $45.7 \%$. Most commonly infection was found in age group of 35 years and older, males, and agricultural workers. Although, the national prevalence may be decreasing but the results show that the $O$. viverrini infection is still high in communities of the northeast region. Therefore, the focus in populations living in northeast Thailand should be screening of infection and changing their eating behavior.
\end{abstract}

Keywords: Opisthorchis viverrini - current status - Thailand

Asian Pac J Cancer Prev, 16 (16), 6825-6830

\section{Introduction}

Opisthorchis viverrini is a trematode parasite that attacks the area of the bile duct including intra and extrahepatic bile ducts. O. viverrini, Southeast Asian liver fluke, is one of the three most medically important species in the family Opisthorchiidae (King and Sholz 2001; Kaewkes 2003). O. viverrini infection is acquired by human, dog, and cats ingest raw or undercooked fish (Sitthithaworn et al., 2003; Kaewpitoon et al., 2007). It causes the disease opithorchiasis and related to a cancer of the gall bladder and/or its ducts; cholangiocarcinoma (CCA) which this CCA is commonly found the high incident in Thailand (Sripa et al., 2007; Kaewpitoon et al., 2008a). In 2009, $O$. viverrini has been classified by the International Agency for Research on Cancer (IARC), World Health Organization (WHO) as a group 1 biological carcinogen in which its capable of causing cancer in human (Sripa et al., 2012). The first human case of $O$. viverrini infection was reported in Thailand (Leiper 1915), to date, it has been reported a serious public health problem in Thailand, Lao People's Democratic Republic, Vietnam, and Cambodia (Yong et a., 2010; Sitthithaworn et al., 2012). Particularly, in the northeast and north region of Thailand, where have been a high incident of $O$. viverrini and CCA (Sripa et al., 2007; Sitthithaworn et al., 2012). Opisthorchiasis has received less attention and it is a neglected disease in Asia, its affect the poor and poorest people (Sripa 2008). In addition, medical care and loss of wages in Thailand costs about \$120 million annually (Muller and Wakelin 2002) or $\$ 120$ million per year can cost Northeast Thailand only (King and Sholz 2001).

Community-based surveys in Thailand have been reported. Update on prevalence and distribution is necessary to summarize, therefore, this review highlights the current status of $O$. viverrini infections in the communities of Thailand. A mini-review was conducted to update the current status of $O$. viverrini in Thailand

${ }^{1}$ Faculty of Public Health, Vongchavalitkul University, ${ }^{2}$ Suranaree University of Technology Hospital, ${ }^{3}$ Parasitic Disease Research, Suranaree University of Technology, ${ }^{5}$ Provincial Public Health of Nakhon Ratchasima, ${ }^{6}$ Nakhon Ratchasima Rajabhat University, Nakhon Ratchasima, ${ }^{4}$ Faculty of Public Health, Khon Kaen University, Khon Kaen, Thailand *For correspondence: natthawut.k@ hotmail.com,natthawut_kae@vu.ac.th 
throughout the active surveillance study. The electronic databases PubMed, MEDLINE, Scopus, Web of Science, and Google Scholar were searched with keywords "Prevalence of Opisthorchis viverrini", "Opisthochis viverrini infection", "current status of Opisthorchis viverrini", "Opisthorchis viverrini in Thailand". The research articles with five years periods from 2010 and 2015 study in human, were included.

\section{Life Cycle and Transmission}

O. viverrini has a freshwater snail as the first intermediate hosts, in which asexual reproduction takes place, and freshwater cyprinid fishes as second intermediate hosts. Humans, dogs and cats, act as definitive hosts, in which sexual reproduction occurs (Young et al., 2010). The first intermediate hosts, freshwater snails (genus Bithynia), ingest the eggs from which the miracidia; asexual, and then a cercaria stage; the free swimming larval stage, is shed from the infected snails. The cercaria penetrates into a cyprinoid fish; the second intermediate host, encysts in the fins, skin and musculature of the fish, and becomes a metacercaria (World Health Organization 1995; Muller and Wakelin 2002; Tohamy and Mohamed 2006; Laha et al., 2007). The second intermediate hosts habitats in the freshwater with stagnant or slow-moving waters mainly ponds, river, aquaculture, swamps, rice fields (Keiser and Utzinger 2007). The metacercariae; the infective stage is ingested by fish-eating mammals; definitive hosts, including human, dogs and cats. Infection is acquired when human ingest raw or undercooked fish. Dishes of raw fish are common in the cuisine of Thailand mainly koi-pla, larb-pla; raw fish in spicy salad, pla-ra, pla som and Som fak; variety of salted semi-fermented fish dishes (World Health Organization 1995; Kaewpitoon et al., 2008). The young adult worm escapes from the metacercarial cyst in the upper small intestine and then migrates through the ampulla of Vater into the biliary tree, where it develops to sexual maturity over four to six weeks, thus completing the life cycle. The lifespan of Opisthorchis viverrini is over 10 years (Harinasuta, and Harinasuta 1984).

\section{Current status of $O$. viverrini infection in Thailand during 2010-2015}

In Thailand, the first nationwide survey of the four regions of Thailand during 1980-1981 revealed an overall prevalence of $O$. viverrini infection of $14 \%$. The highest of infection was found in the Northeast (34.6\%), and flowed by the Central $(6.3 \%)$, the North $(5.6 \%)$ and the South $(0.01 \%)$ regions (Jongsuksuntigul and Imsomboon 2003; Sithithaworn et al., 2012). Since then, the average national prevalence of infection has declined to $8.7 \%$ in the year 2009 , with the intensive and continuous control programs and public health service activities. This figure indicates that more than 6 million are infected with $O$. viverrini in Thailand, particularly in the north and northeast regions (Sithithaworn et al., 2012). The prevalence of $O$. viverrini infection remains high in various parts of the country, especially in northeast Thailand and particularly in wetland rural areas where a large proportion of the community work in agriculture and continue the traditional practice of eating raw or undercooked cyprinoid fish products. The national control program seems to have had little impact in many of these areas, and it has been difficult to make precise assessments of the overall effectiveness of the program (Sripa et al., 2007; Sitthithaworn et al., 2012).

In this review highlight the current prevalence of $O$. viverrini in the communities of Thailand during five years period 2010-2015, data is shown in Table 1. No survey of $O$. viverrini infection in 2010 in which search through out electronic standard database included Thai-TCI. In year 2011, 2 articles have been reported. A descriptive crosssectional study was carried out in Khon Kaen province, northeast region. There were 338 sample subjects aged between 20-60 years, from urban, semi-urban and rural areas. Almost one third (32\%) reported testing positive for O. viverrini eggs in stool (Promthet et al., 2011). The border provinces included Nan in the north, Ubon Ratchathani and Khon Kaen in the northeast, were collected stool samples and examinations. The helminthic infections were examined by using Kato thick-smear technique. Among a population sample of 109 males and 143 females in lowland Nan, with ages ranging between 15-85 years, $74(29.4 \%)$ tested positive for the presence of Opisthorchis-like eggs. The results of the study in highland Nan were very different: Opisthorchis-like eggs were only found in one subject $(0.5 \%)$ out of the 223 hilltribe members. In Ubon Ratchathani, population sample of 144 males and 152 females with ages ranging between 15-82 years, $19(6.4 \%)$ tested positive for the presence of Opisthorchis-like eggs. In Khon Kaen, population sample of 108 males and 113 females with ages ranging between 15-76 years, $16(7.2 \%)$ tested positive for the presence of Opisthorchis-like eggs, respectively.

In 2012, a total of 7 community-based surveys was reported. Of 435 stool samples from Mahasarakham province was prepared by formalin-ether concentration technique and examined. $5.74 \%$ of subject was infected with $O$. viverrini (Thanchomnang et al., 2012). Active surveillance on opisthorchiasis was performed in 8,936 males and females aged from 20 to 60 years from 90 villages of Khon Kaen province, northeast Thailand. All were stool-examined for $O$. viverrini infection by standard quantitative formalin/ethyl acetate concentration technique. 3,359 participants $(37.59 \%)$ with stool $O$. viverrini egg positive, this study emphasizes the current status of high $O$. viverrini infection rate. Of these participants with stool egg positive underwent ultrasonography of the upper abdomen for CCA surveillance (Mairiang et al., 2012). The cross-sectional study in a prospective cohort study was to examine the prevalence and co- infection of intestinal parasites among northeastern Thai rural residents, recruited into the Khon Kaen Cohort Study (KKCS), and who were residing in areas of high-risk for developing CCA. On recruitment, subjects testing using the formalin ethyl-acetate concentration technique. 18,900 of cohort subjects, and $45.7 \%$ were found to be positive for $O$. viverrini infection. According to a mapping analysis, a higher CCA burden was correlated with a higher prevalence and a greater intensity of $O$. viverrini 
Opisthorchis viverrini Infection - Review and Current Status at the Community Level in Thailand

(Songserm et al., 2012). A prospective research project designed to monitor the impact of the national control program in rural communities located in a northeastern province and at high risk of $O$. viverrini infection. 1,569 villagers, aged 20-65 years, living in Yasothon province were examined and found that $38.68 \%$ were infected with $O$. viverrini. Males were slightly more likely to be infected than females, and the infection was found to be positively associated with age in both males and females (Saengsawang et al., 2012). In lower northeast was performed, a community-based cross-sectional survey of $O$. viverrini infection was conducted among 333 elderly in 17 districts of Surin province, during one year period from January to December 2011. O. viverrini infection was determined using Kato's Thick Smear technique. Overall intestinal parasitic infection was $16.2 \%$, predominantly in $O$. viverrini $(9.91 \%)$. The $O$. viverrini infection was found higher in males $(13.8 \%)$ than females $(7.83 \%)$, and frequently in elderly 60-70 year old with $14.2 \%$ (Kaewpitoon et al., 2012). In addition, Kaewpitoon et al., (2012) have been reported the prevalence of $O$. viverrini infection in Nakhon Ratcahsima where is located near Surin province. A cross-sectional survey was conducted during a one year period from October 2010 to September 2011, the infection was determined using a modified Kato's thick smear technique. A total of 1,168 stool samples were obtained from 516 males and 652 females, aged 5-90 years. Stool examination showed that $2.48 \%$

Table 1. Community-based Surveys of $O$. viverrini Infection in Thailand During 2010-2015, by Study Design, Diagnostic Method, Number of Case, Prevalence, and Investigator

\begin{tabular}{|c|c|c|c|c|}
\hline Study design & Method & No. of cases & Prevalence & Investigators \\
\hline Case-control study & Modified Kato-Katz technique & 351 & 33.3 & $\begin{array}{l}\text { Chudthaisong et al. } \\
2015\end{array}$ \\
\hline Cross-sectional analytic study & Modified Kato-Katz technique & 254 & 15 & Chaiputcha et al. 2015 \\
\hline Cohort study & $\begin{array}{c}\text { Modified formalin/ethyl acetate } \\
\text { concentration technique /indirect } \\
\text { ELSIA }\end{array}$ & 469 & 7 & Yeoh et al., 2015 \\
\hline Cross-sectional study & Modified Kato Katz method & 3,916 & 22.7 & $\begin{array}{l}\text { Thaewnongiew et al., } \\
2014\end{array}$ \\
\hline Cross-sectional study & $\begin{array}{l}\text { Formalin-ethyl acetate concentration } \\
\text { methods }\end{array}$ & 253 & 26.9 & $\begin{array}{l}\text { Boonjaraspinyo et al., } \\
2013\end{array}$ \\
\hline Cross-sectional study & Kato thick smear technique & 684 & 37.2 & $\begin{array}{l}\text { Saengsawang et al., } \\
2013 \\
\end{array}$ \\
\hline $\begin{array}{c}\text { Community-based prospective } \\
\text { Cohort study }\end{array}$ & $\begin{array}{l}\text { Kato and formalin-ethyl acetate } \\
\text { concentration technique }\end{array}$ & 1,204 & 18.6 & $\begin{array}{l}\text { Suwannahitatorn et al., } \\
2013\end{array}$ \\
\hline Cross-sectional survey & Formalin-ether concentration technique & 331 & 13.9 & Yahom et al., 2013 \\
\hline Cross-sectional survey & Modified Kato's thick smear technique & 1,168 & 2.48 & $\begin{array}{c}\text { Kaewpitoon et al., } \\
2012\end{array}$ \\
\hline $\begin{array}{l}\text { Community-based cross- } \\
\text { sectional survey }\end{array}$ & Kato's Thick Smear technique & 333 & 9.91 & $\begin{array}{l}\text { Kaewpitoon et al., } \\
2012 \\
\end{array}$ \\
\hline Cross-sectional study & Kato's Thick Smear technique & 1,569 & 38.7 & $\begin{array}{l}\text { Saengsawang et al., } \\
2012\end{array}$ \\
\hline Cross-sectional study & $\begin{array}{c}\text { Formalin-ether sedimentation and } \\
\text { high annealing temperature random } \\
\text { amplified polymorphic DNA PCR } \\
\text { methods }\end{array}$ & 316 & 6.54 & $\begin{array}{l}\text { Wongsawad et al., } \\
2012\end{array}$ \\
\hline $\begin{array}{l}\text { Cross-sectional study in a } \\
\text { prospective cohort study }\end{array}$ & $\begin{array}{l}\text { Formalin ethyl acetate concentration } \\
\text { technique }\end{array}$ & 18,900 & 45.7 & Songserm et al., 2012 \\
\hline Cross sectional study & $\begin{array}{l}\text { Formalin/ethyl acetate concentration } \\
\text { technique }\end{array}$ & 8,936 & 41.5 & Mairiang et al., 2012 \\
\hline Cross sectional study & Formalin-ether concentration technique & 435 & 5.74 & $\begin{array}{l}\text { Thanchomnang et al., } \\
2012\end{array}$ \\
\hline $\begin{array}{l}\text { Descriptive cross-sectional } \\
\text { study }\end{array}$ & Kato's Thick Smear technique & 338 & 32 & Promthet et al., 2011 \\
\hline Cross sectional study & Kato-Katz technique & $\begin{array}{c}\text { Nan }(475)^{*} \\
\text { Khon Kane } \\
(221) \\
\text { Ubon } \\
\text { Ratchathani } \\
\quad(296)\end{array}$ & $\begin{array}{c}15.79 \\
7.2 \\
6.4\end{array}$ & $\begin{array}{l}\text { Anantaphruti et al., } \\
2011\end{array}$ \\
\hline
\end{tabular}


Natthawut Kaewpitoon et al

Table 2. Community-based Surveys of O. viverrini Infection in Thailand during 2010-2015, by Age, Gender, Occupation, and Location of Survey, Investigator

\begin{tabular}{|c|c|c|c|c|}
\hline Study survey (province) & Age & Gender & Occupation & Investigators \\
\hline Nong Khai & $\begin{array}{c}35-54 \\
>54\end{array}$ & Male & Agriculture & Chudthaisong et al. 2015 \\
\hline Mahasarakham & $\begin{array}{c}30-44 \\
45-59 \\
>59\end{array}$ & Male & Agriculture & Chaiputcha et al. 2015 \\
\hline Khon Kaen & $35-44$ & - & - & Yeoh et al., 2015 \\
\hline Nakhonphanom Sakonnakhon Nongkhai & $40-49$ & Male & & Thaewnongiew et al., 2014 \\
\hline Khon Kaen & $>60$ & Male & & Boonjaraspinyo et al., 2013 \\
\hline Yasothon & $20-35$ & Male & Government service & Saengsawang et al., 2013 \\
\hline Chachengsao & $\begin{array}{c}25-59 \\
>59\end{array}$ & Male & Agriculture & Suwannahitatorn et al., 2013 \\
\hline MahasarakhamKalasin & $>45$ & Male & Agriculture & Yahom et al., 2013 \\
\hline Nakhon Ratchasima & $51-60$ & Male & Agriculture & Kaewpitoon et al., 2012 \\
\hline Surin & $60-70$ & Male & Agriculture & Kaewpitoon et al., 2012 \\
\hline Yasothon & $\begin{array}{l}36-55 \\
56-65\end{array}$ & Male & Agriculture & Saengsawang et al., 2012 \\
\hline Chiang Mai & - & - & - & Wongsawad et al., 2012 \\
\hline Khon Kaen Cohort Study (KKCS) & - & Male & - & Songserm et al., 2012 \\
\hline Khon Kaen & $40-49$ & Male & Agriculture & Mairiang et al., 2012 \\
\hline Mahasarakham & - & Male & - & Thanchomnang et al., 2012 \\
\hline Khon Kaen & - & - & - & Promthet et al., 2011 \\
\hline Nan, Khon Kaen, Ubonratchathani & - & - & - & Anantaphruti et al., 2011 \\
\hline
\end{tabular}

were infected with $O$. viverrini. Males were slightly more likely to be infected than females, but the different was not statistically significant. Positive results were evident in 16 of 32 districts, the highest prevalence being found in Non Daeng with $16.7 \%$, followed by Pra Thai with $11.1 \%$, Kaeng Sanam Nang with $8.33 \%$, and Lam Ta Men Chai $(8.33 \%)$ districts. A high throughput method was employed to identify mixed infection between $O$. viverrini and minute intestinal fluke in the north region. 316 fecal specimens of people living along the Mae Ping River, Chomtong district, Chiangmai province, were examined seasonally for trematode eggs using a compound microscope. Positive cases were analyzed in HAT-RAPD, DNA profiles were compared with adult stages to determine the actual species infected, and specific DNA markers of each fluke were also screened. The results showed that $6.54 \%$ of $O$. viverrini was found only in the hot-dry season. This study demonstrates the mixed infection of $O$. viverrini and Haplorchis taichui and confirms the extended distribution of $O$. viverrini in northern Thailand (Wongsawad et al., 2012).

In 2013, O. viverrini infection was examined in 331 stool samples from Maha Sarakham and Kalasin province where located in the upper northeast region, by formalinether concentration technique. $O$. viverrini infection $(13.90 \%)$ was the most common pathogenic intestinal parasitic infection, followed by Strongyloides stercoralis $(11.47 \%)$ and Taenia sp (1.81\%) (Yahom et al., 2013). A prospective research project to monitor the impacts of a national liver fluke control program in a rural community of northeast Thailand, a sample of 684 villagers aged 20-65 years was tested for infection using the Kato thick smear technique. The overall prevalence of $O$. viverrini infection was $37.2 \%$ and was highest in the 20-35 year age group. As many as $91.8 \%$ reported eating fish dishes known to place them at risk of infection (Saengsawang et al., 2013). The village scale in Khon Kaen province, northeastern Thailand was surveyed from March to August 2013. A total of 253 stool samples from 102 males and 140 females, aged 2-80 years, were prepared using formalin-ethyl acetate concentration methods. The most common parasite found was $O$. viverrini (26.9\%), followed by S. stercoralis (9.5\%), Taenia spp. (1.6\%), Echinostomes (0.4\%), and hookworms (0.4\%) (Boonjaraspinyo et al., 2013). While, one study in central region was conducted. A prospective cohort study was conducted to evaluate the incidence of $O$. viverrini infection in Chachengsao province by using Kato and formalin-ethyl acetate concentration technique. The results indicate that the prevalent and incident of $O$. viverrini infection were $18.6 \%$ and $21.4 \%$, respectively. Consumption of chopped raw fish salad, Koi pla and age 60 years and older were independently associated with $O$. viverrini infection (Suwannahitatorn et al., 2013).

In 2014, an investigation of prevalence and risk factors for opisthorchiasis in 3,916 Thai residents of Nakhon Phanom, Sakon Nakhon, and NongKhai province who were 15 or over, and stool examinations for parasite eggs (using the Modified Kato Katz method) was reported. The prevalence of opisthorchiasis was $22.7 \%$. The province with the highest prevalence was Nakhorn Phanom (40.9\%; 
female to male ratio $=1: 1.2$ ). The age group with the highest prevalence was 40-49 year olds. All age groups had a prevalence $>20 \%$ (Thaewnongiew et al., 2014). In 2015, the prevalence of $O$. viverrini infection in the cohort subjects (as well as new subjects) was investigated using faecal egg counts. O. viverrini infection rates in the same individuals of the cohort were lower in 2006 than in 1992 , was $7.0 \%$. Also, by studying the period effect, the current $35-44$ year olds had a $12.4 \%$ (95\% CI $3.9 \%$ to $20.9 \%$ ) lower prevalence of $O$. viverrini infection than the $35-44$ year olds in 1992 (24.2\% versus $11.8 \%$ ) (Yeoh et al., 2015). The cross-sectional analytic study was to investigate the prevalence for $O$. viverrini infection in 254 household representative aged 15 years or older living in the most urbanized part of Chiang Yuen municipality in Mahasarakham province, northeastern Thailand. All participants provided stool samples in which were examined using the modified Kato-Katz procedure. The overall prevalence of $O$. viverrini infection was $15.0 \%$, and in the multivariate analysis male gender was found to be significantly and positively associated with $O$. viverrini infection (ORadj=9.75, 95\%CI: 34.03-23.58) while education to secondary school level or above was a significant protective factor $(\mathrm{ORadj}=0.30,95 \% \mathrm{CI}$ : 0.12-0.74) (Chaiputcha et al. 2015). Among 516 people in Nong Khai province in July 2013 was examined $O$. viverrini infection. Stool specimens were examined for intestinal parasites within hours after collection using a normal saline wet preparation and the modified Kato- Katz technique. Of $117(22.67 \%)$ participants have a positive test result for $O$. viverrini infection (Chudthaisong et al., 2015)

The $O$. viverrini infection was found in age between 20-80 year old, and the most frequently in 35-55 year old. Moreover, older people are still found high infection, particularly in age over 60 year old. Infection in gender was characterized and found that male was slightly found more than female. In addition, occupation with agriculture was the most frequently $O$. viverrini infection. Characteristic data are shown in Table 2.

\section{Conclusions}

The results show that the prevalence of $O$. viverrini infections in Thailand is still high, particularly in the northeast region. Proactive education about dietary habits, personal hygiene, and sanitation should be provided to the people in the rural community to reduce the prevalence of $O$. viverrini infections. In order to reduce the prevalence of opisthorchiasis, the focus in populations living in Northeast Thailand should be changing their eating behaviors as appropriate to their tradition and context.

\section{References}

Anantaphruti MT, Maipanich W, Kusolsuk T, et al (2011). Taeniasis and other helminthic infections in the northern and northeastern border provinces of Thailand. J Trop Med Parasito, 3, 62-9.

Boonjaraspinyo S, Boonmars T, Kaewsamut B, et al (2013). A cross-sectional study on intestinal parasitic infections in rural communities, northeast Thailand. Korean J Parasitol, 51, 727-34.

Chaiputcha K, Promthet S, Bradshaw P (2015). Prevalence and risk factors for infection by Opisthorchis viverrini in an urban area of mahasarakham province, northeast Thailand. Asian Pac J Cancer Prev, 16, 4173-6.

Chudthaisong N, Promthet S, Bradshaw P (2015). Risk factors for Opisthorchis viverrini infection in Nong Khai province, Thailand. Asian Pac J Cancer Prev, 16, 4593-6.

Harinasuta C, Harinasuta T (1984). Opisthorchis viverrini: Life cycle, intermediate hosts, transmission to man and geographical distribution in Thailand. ArzneimittelForschung, 34, 1164-7.

Jongsuksuntigul P, Imsomboon T (2003). Opisthorchiasis control in Thailand. Acta Trop, 88, 229-32.

Kaewkes S (2003). Taxonomy and biology of liver flukes. Acta Trop, 88, 177-86.

Kaewpitoon N, Kaewpitoon SJ, Pengsaa P, et al (2007). Knowledge, attitude and practice related to liver fluke infection in northeast Thailand. World J Gastroenterol, 13, 1837-40.

Kaewpitoon N, Kaewpitoon SJ, Pengsaa P, Sripa B (2008). Opisthorchis viverrini: the carcinogenic human liver fluke. World J Gastroenterol, 14, 666-74.

Kaewpitoon N, Kaewpitoon SJ,Pengsaa P(2008). Opisthorchiasis in Thailand: review and current status. World J Gastroenterol, 14, 2297-302.

Kaewpitoon SJ, Rujirakul R, Ueng-Arporn N, et al (2012). Community-based cross-sectional study of carcinogenic human liver fluke in elderly from Surin province, Thailand. Asian Pac J Cancer Prev, 13, 4285-8.

Kaewpitoon SJ, Rujirakul R, Kaewpitoon N (2012). Prevalence of Opisthorchis viverrini infection in Nakhon Ratchasima province, Northeast Thailand. Asian Pac J Cancer Prev, 13, 5245-9.

Keiser J, Utzinger JR (2007). Artemisinins and synthetic trioxolanes in the treatment of helminth infections". Curr Opin Infect Dis, 20, 605-12.

King S, Scholz, TS (2001). Trematodes of the family Opisthorchiidae: A minireview. Korean J Parasitol, 39, 209-21.

Laha T, Pinlaor P, Mulvenna J, et al (2007). Gene discovery for the carcinogenic human liver fluke, Opisthorchis viverrini. BMC Genom, 8, 189.

Leiper RT (1915). Notes of the occurence of parasites presumably rare in man in man. $J$ R Army Med Corps, 24, 569-75.

Mairiang E, Laha T, Bethony JM, et al (2012). Ultrasonography assessment of hepatobiliary abnormalities in 3359 subjects with Opisthorchis viverrini infection in endemic areas of Thailand. Parasitol Int, 61, 208-11.

Muller R, Wakelin D (2002). Worms and human disease. 2ed. CAB International; UK, 33-44.

Promthet S, Saranrittichai K, Kamsa-ard S, et al (2011). Situation analysis of risk factors related to non-communicable diseases in Khon Kaen Province, Thailand. Asian Pac J Cancer Prev, 12, 1337-40.

Saengsawang P, Promthet S, Bradshaw P (2012). Prevalence of OV infection in Yasothon province, northeast Thailand. Asian Pac J Cancer Prev, 13, 3399-402.

Saengsawang P, Promthet S, Bradshaw P (2013). Infection with Opisthorchis viverrini and use of praziquantel among a working-age population in northeast Thailand. Asian Pac J Cancer Prev, 14, 2963-6.

Sithithaworn P, Haswell-Elkins M (2003). Epidemiology of Opisthorchis viverrini. Acta Trop, 88, 187-94.

Sithithaworn P, Andrews RH, Nguyen VD, et al (2012). The current status of opisthorchiasis and clonorchiasis in the 
Mekong Basin. Parasitol Int, 61, 10-6.

Sithithaworn P, Andrews RH, Nguyen VD, et al (2012). The current status of opisthorchiasis and clonorchiasis in the Mekong Basin. Parasitol Int, 61, 10-6.

Songserm N, Promthet S, Wiangnon S, et al (2012). Prevalence and co-infection of intestinal parasites among thai rural residents at high-risk of developing cholangiocarcinoma: a cross-sectional study in a prospective cohort study. Asian Pac J Cancer Prev, 13, 6175-9.

Sripa B, Kaewkes S, Sithithaworn P, et al (2007). Liver fluke induces cholangiocarcinoma. PLoS Medicine, 4, 201.

Sripa, B. (2008). Concerted action is needed to tackle liver fluke infections in Asia. PLoS Neg Trop Dis, 2, 232.

Sripa B, Brindley PJ, Mulvenna J, et al (2012). The tumorigenic liver fluke Opisthrochis viverrini-multiple pathways to cancer. Trend Parasitol, 28, 395-407.

Suwannahitatorn P, Klomjit S, Naaglor T, Taamasri P, et al (2013). A follow-up study of Opisthorchis viverrini infection after the implementation of control program in a rural community, central Thailand. Parasit Vectors, $6,188$.

Thaewnongiew K, Singthong S, Kutchamart S, et al (2014). Prevalence and risk factors for Opisthorchis viverrini infections in upper Northeast Thailand. Asian Pac J Cancer Prev, 15, 6609-12.

Thanchomnang T, Yahom S, Radomyos P (2012). Prevalence of Intestinal Parasitic Infections in Villagers and Teachers in Mahasarakham Province. J Trop Med Parasitol, 35, 63-7.

Tohamy AA, Mohamed SM (2006). Chromosomal studies on two Egyptian freshwater snails, cleopatraand bithynia (molluscaprosobranchiata). Arab J Biotech, 9, 17-26.

Wongsawad C, Phalee A, Noikong W, et al (2012). Co-infection with Opisthorchis viverrini and haplorchis taichui detected by human fecal examination in chomtong district, Chiang Mai Province, Thailand. Parasitol Int, 61, 56-9.

World Health Organization (1995). Control of foodborne trematode infection. WHO Technical Report Series, 849, 89-91.

Yahom S, kullawat J, Boonsai D, et al (2013). The Prevalence of intestinal parasitic infections in Maha Sarakham and Kalasin provinces. J Trop Med Parasitol, 36, 29-33.

Young ND, Campbell BE, Hall RS, et al (2010). Unlocking the transcriptomes of two carcinogenic parasites, Clonorchis sinensis and Opisthorchis viverrini. PLoS Neg Trop Dis, 4, 719 .

Yeoh KW, Promthet S, Sithithaworn P, et al (2015). Reexamination of Opisthorchis viverrini infection in northeast Thailand. Asian Pac J Cancer Prev, 16, 3413-8. 